'Instituto de Neurociencias Clínicas, Universidad Austral de Chile. Valdivia, Chile. 2Departamento de Psiquiatría, Escuela de Medicina, Pontificia Universidad Católica de Chile. Santiago, Chile.

${ }^{3}$ Instituto Milenio para la Investigación de la Depresión y Personalidad. Santiago, Chile. ${ }^{4}$ Centro de Educación Médica y Ciencias de la Salud, Facultad de Medicina, Pontificia Universidad Católica de Chile, Santiago, Chile. Instituto de Estudios

Psicológicos, Universidad Austral de Chile. Valdivia, Chile.

${ }^{6}$ Centro Interdisciplinario de Estudios del Sistema Nervioso, Universidad Austral de Chile. Valdivia, Chile.

${ }^{7}$ Núcleo Milenio para mejorar la Salud Mental de Adolescentes y Jóvenes (Imhay). Santiago, Chile. aPsicólogo. bPhD.

Correspondencia a: Dr. Álvaro Langer Instituto de Estudios Psicológicos, Facultad de Medicina, Campus Isla Teja, 5090000 Valdivia. alvaro.langer@uach.cl

\title{
Mindfulness y la relación clínica: pasos hacia una resiliencia en medicina
}

\author{
EDWIN KROGH ${ }^{1}$, SEBASTIÁN MEDEIROS ${ }^{2,3}$, \\ MARCELA BITRAN ${ }^{4}$, ÁLVARO I. LANGER ${ }^{3,5,6,7, a, b}$
}

\section{Mindfulness and the clinical relationship: steps to a resilience in medicine}

\begin{abstract}
Medical resilience is a dynamic capacity, which has the potential to improve the well-being of physicians and to enhance the quality of the clinical relationship. Strategies to promote resilience are important to achieve a sustainable medical practice and improve patient care. Mindfulness training has demonstrated to be an effective tool to promote resilience in physicians. This paper contextualizes the place of mindfulness in medical practice and describes the ways through which it can contribute to resilience in medicine. The concept of mindfulness, its relationship with health practice is reviewed and the benefits of the practice of mindfulness in the clinical relationship are described. We suggest that the benefits achieved through a mindfulnessbased medical practice are mediated by two axes. One is the nonspecific and specific effect of mindfulness-based practices and the other is the integration of explicit and implicit knowledge of clinical practice. We conclude that medical practice that integrates mindfulness can contribute to the challenge of achieving greater levels of individual, staff and institutional resilience. There is a need to have continuing mindfulness training programs for health professionals and to integrate this concept in the curriculum of health care professionals.
\end{abstract}

(Rev Med Chile 2019; 147: 618-627)

Key words: Empathy; Mindfulness; Psychological Techniques; Resilience; Psychological.

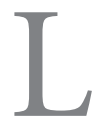
a resiliencia es la capacidad de un individuo para adaptarse y manejar el estrés y la adversidad ${ }^{1}$. En las últimas dos décadas la idea de 'resiliencia médica' se ha instalado en la investigación sobre la práctica clínica. La resiliencia médica ha sido descrita como una sinergia entre características personales, tales como autovaloración, flexibilidad cognitiva y relacional, habilidades de organización, asertividad y humor, y la disponibilidad de recursos profesionales y organizacionales, tales como apoyo administrativo, capacidad de trabajo en equipo y disponibilidad de insumos ${ }^{2}$. Se ha sugerido que las estrategias asociadas a la resiliencia determinan la diferencia entre médicos que experimentan estrés y burnout por la exposición continua a situaciones estresantes y aquellos que pueden adaptarse a estas exigencias del contexto clínico sin deterioro en su bienestar ${ }^{3}$. La resiliencia es una capacidad dinámica, que tiene el potencial de mejorar el bienestar de los médicos y de favorecer la calidad de la atención ${ }^{4}$. Estrategias para promover la resiliencia son clave para promover una práctica médica sostenible y mejorar la atención al paciente ${ }^{5}$.

Sin embargo, surge el desafío de identificar modos de afrontamiento de la práctica clínica que promuevan la resiliencia, que reduzcan el riesgo de burnout y que redunden en una mayor satisfacción por parte del clínico y del paciente ${ }^{6}$. Existen numerosas experiencias que buscan promover la 
resiliencia médica, mejorar la calidad de atención y el autocuidado en Medicina ${ }^{7-9}$. Entre ellas, el entrenamiento en mindfulness ha demostrado ser una herramienta efectiva ${ }^{10-12}$. Por ejemplo, en un clásico trabajo, Krasner y su equipo demostraron que los médicos que participaron en un programa de entrenamiento basado en mindfulness lograron un mayor bienestar personal y el desarrollo de actitudes centradas en el paciente, tales como empatía y compasión, tanto a corto plazo como sostenidas en el tiempo ${ }^{10}$.

El objetivo del presente trabajo es contextualizar el sitial del mindfulness en la práctica médica y describir las vías a través de las cuales éste puede contribuir a la resiliencia en Medicina. Para ello se revisará el concepto de mindfulness y su relación con la práctica en salud, para luego describir los beneficios de la práctica del mindfulness y las formas para lograr mayores niveles de resiliencia en Medicina.

\section{Concepto de mindfulness y su relación con la práctica médica}

Mindfulness o Atención Plena es una práctica enraizada en varias tradiciones contemplativas, tanto religiosas como seculares. Ha sido parte del hinduismo, budismo y del yoga y, más recientemente, de la meditación secular. El mindfulness que ha sido incorporado como práctica secular en occidente encuentra sus fundamentos en las tradiciones contemplativas de la psicología budista ${ }^{13}$.

Mindfulness puede ser entendido como la práctica de regresar una y otra vez a la mantención de un estado de completa atención a lo que se va desplegando en el momento presente, tanto de la experiencia externa como interna y acompañado por una actitud no enjuiciadora de dicha experiencia. Es la capacidad de estar completamente presente, consciente de cómo se está, qué se está haciendo o qué está sucediendo, acompañada de una baja tendencia a la reactividad emocional. Alude a aceptar y abrirse a todas las dimensiones de la experiencia, sean experimentadas como agradables, neutras o desagradables ${ }^{14-16}$.

La popularidad alcanzada por el mindfulness tiene, posiblemente, un doble origen. Por una parte, está el programa de reducción del estrés basada en la atención plena (MBSR, Mindfulness-Based Stress Reduction, por sus siglas en in- glés), un modelo de intervención desarrollado por Jon Kabat-Zinn a finales de la década de 1970, como una terapia complementaria para personas con problemas médicos, a partir de la cual se ha desarrollado un amplio espectro de modalidades terapéuticas ${ }^{17,18}$.

Por otra, prominentes científicos y clínicos se han familiarizado con el concepto de mindfulness a partir de las reuniones organizadas por el Mind and Life Institute desde 1987, que promueven diálogos formales y regulares entre el Dalai Lama y científicos y profesionales de varias disciplinas ${ }^{19}$. Adicionalmente, el mindfulness se ha transformado en una relevante área de estudio y de intervención en psicología ${ }^{20}$, psiquiatría ${ }^{21}$ medicina $^{22}$, educación ${ }^{23}$ y recursos humanos, ${ }^{24}$ entre otros.

El ámbito en el que el mindfulness ha encontrado especial arraigo en las últimas décadas es en las ciencias de la salud. Se han desplegado tres áreas a través de las cuales la práctica de la atención plena ha sido implementada en la atención en salud: 1) como modelo de intervención para atención de pacientes ${ }^{25,26} ; 2$ ) para favorecer la calidad de la atención ${ }^{27-29}$ y 3) como estrategia de autocuida$\mathrm{do}^{30,31}$. El presente artículo apunta principalmente las dos últimas áreas.

Desde el pionero artículo Mindful Practice de Ronald Epstein ${ }^{32}$ en 1999, el interés y la aplicación del mindfulness en la práctica médica ha crecido de forma sostenida. Actualmente, numerosos centros clínicos universitarios, públicos y privados ofrecen programas de formación en mindfulness tanto en pregrado como en postgrado para médicos y profesionales afines ${ }^{33(\mathrm{p} .43), 34}$.

La incorporación de la práctica del mindfulness en atención en salud como estrategia de autocuidado supone una paradoja que ha desafiado a la medicina. Por una parte, el foco de la práctica del mindfulness no apunta a la reducción sintomática. La intención central del mindfulness no apunta a la reducción sintomática, es decir, despojarse del síntoma, del dolor o el distrés asociado a una condición de salud o a la práctica médica. El objetivo de las prácticas contemplativas en salud busca transformar el impacto del síntoma en las personas, al modificar el modo cómo ésta percibe y experimenta lo que está viviendo ${ }^{35,36}$. Por otra parte, la incorporación de intervenciones basadas en mindfulness (IBM) implica procesos psiconeurobiológicos complejos ${ }^{37}$. En población general, los estudios actuales intentan dilucidar 
qué prácticas contemplativas conducen a qué habilidades específicas y de forma diferenciada ${ }^{38}$, ya sea a través del cultivo de la presencia y consciencia corporal, el desarrollo de la toma de perspectiva y empatía, y el cultivo de actitudes compasivas. Cada forma implica cambios neurofisiológicos y psicológicos particulares. Por ejemplo, en la regulación emocional basada en mindfulness, habría una circularidad entre mecanismos 'top down'-procesos cognitivos en primer plano (de-reificación, defusión, resignificación, etc.)- y mecanismos 'bottom up'-donde el dar espacio a la experiencia somática del momento presente es el foco principal de atención ${ }^{39}$.

\section{Beneficios de una práctica médica basada en mindfulness}

\section{Satisfacción del paciente}

Tanto pacientes como sus familiares suelen responder favorablemente a modelos de atención clínica basados en mindfulness y compasión ${ }^{28,33}$.

Las personas valoran más la experiencia inmediata y cotidiana con el médico u otro profesional de la salud que los resultados que dicho encuentro en salud puede lograr ${ }^{40-43}$. La capacidad de estar presente en el acto clínico de un modo terapéutico ha sido asociada a una mejor alianza terapéutica entre médico y paciente ${ }^{44}$. Asimismo, la satisfacción del paciente y su familia es la respuesta natural a una atención en la que el médico se encuentra atento y despliega una actitud genuinamente interesada en la situación del paciente ${ }^{45}$.

\section{Habilidades comunicacionales}

A través de una favorable comunicación, la relación médico-paciente puede desplegarse de forma efectiva ${ }^{44-46}$. Los pacientes suelen detectar cuando un médico no atiende o no se comunica de forma adecuada ${ }^{45}$, lo que redunda en un deterioro de la adherencia al tratamiento ${ }^{47}$.

Una actitud clínica que incorpora el mindfulness logra que el médico mantenga el foco de atención sobre la interacción con el paciente y no sea arrastrado por pensamientos y emociones que surgen en el momento. También es posible que un mayor nivel atencional del médico permita una mayor "economía comunicacional", evitando preguntas inoficiosas o redundantes. También puede contribuir a reducir el riesgo de falta de tacto y sesgo implícito ${ }^{48}$.

\section{Estrés y burnout}

Actitudes deficientes en autocuidado se encuentran en el corazón de la cultura médica. Existe amplia evidencia que el estrés crónico y burnout (entendido como un síndrome de agotamiento físico y mental relacionado con la vida laboral $)^{49}$, acompañan a la profesión médica desde el pregrado ${ }^{50-53}$. Es posible identificar múltiples factores explican el mantenimiento de estrés y burnout entre los médicos. Éstas suelen interactuar entre sí favoreciendo su aparición. Entre estos se encuentran la carga asistencial, deterioro en las relaciones laborales, los registros electrónicos e institucionales o la infraestructura deficiente, así como perfeccionismo, autocrítica, deficiente regulación emocional o presencia de problemática personal ${ }^{53}$.

Muchos modelos de intervención han demostrado ser útiles al momento de reducir y prevenir el estrés y burnou ${ }^{54,55}$. Existe evidencia que sugiere que la incorporación de entrenamiento basado en mindfulness a médicos contribuye a reducir los síntomas asociados al burnout ${ }^{56-59}$. Es así como, por un lado, el cultivo de la atención plena puede favorecer que el médico logre ser más consciente de su propio estrés y las fuentes de las que surge y, por el otro, puede contribuir a que logre desarrollar estrategias para prevenir el estrés y desplegar la apropiada cantidad de esfuerzo en las actividades que desarrolla. Asimismo, participantes del área de la salud que han participado en IBM describen que observan una reactividad reducida frente a eventos estresantes y que tienden a recuperarse más rápido y de mejor forma frente a ellos ${ }^{10,60}$. Estas características se encuentran en el centro de las habilidades de resiliencia.

\section{Razonamiento clínico, toma de decisiones y prevención de errores}

Estados en los que predomina la fatiga, distracción, ansiedad, enojo, la multitarea o irritabilidad son frecuentes de encontrar en la práctica médica cotidiana $^{33(\text { p.37) }}$. Estos estados pueden conducir a un deterioro en el razonamiento clínico y favorecer errores clínicos. En estos estados se reduce la capacidad de atención y de procesamiento cognitivo. La ansiedad o la prisa ante situaciones clínicas complejas puede favorecer sesgos cognitivos, pérdida de información relevante y una conducta evitativa ${ }^{61,62(\mathrm{p} .22)}$.

El mindfulness puede contribuir a tomar mayor 
consciencia del propio estado físico y emocional, reconociendo estados de fatiga o irritabilidad, para así tomar decisiones que prevengan consecuencias negativas. El cultivo de la atención plena puede reducir la tendencia a un funcionamiento automatizado, limitando los sesgos cognitivos que naturalmente emergen en la práctica clínica. Por ejemplo, Borrell-Carrió y Epstein señalan que cuando los médicos se enfrentan al desafío de resolver una situación clínica, experimentan un grado de tensión emocional. Esta tensión es mayor cuando la situación es atípica o ambigua, o cuando el paciente es percibido como exigente. Estos autores plantean que el riesgo de error clínico se reduce si el médico es capaz de ser consciente y de tolerar la tensión emocional que el 'no saber' genera $^{63}$.

\section{Empatía y compasión}

Una de las dimensiones que se encuentran en el corazón de los beneficios de una práctica médica sustentada en el mindfulness es el desarrollo de actitudes empáticas y compasivas. Pese a ser actitudes altamente valoradas por pacientes y sus familias, son deficientemente entrenadas en pregrado y casi nulamente reforzadas durante la vida profesional. Cultivar la empatía y compasión en medicina no es fácil. Son muy diversos los factores que contribuyen a que actitudes empáticas y compasivas se agoten ${ }^{64-70}$. Entre muchos otros, destacan la idea de trauma vicario $^{66}$, exposición prolongada a estrés ${ }^{64,65}$, historia y características personales ${ }^{67,68}$ y estar en la primera línea de atención ${ }^{66,67}$. El estrés crónico favorece la tendencia a conductas de autoprotección, paralización o ataque ${ }^{71}$. Conductas que, a su vez, son capaces de interferir negativamente en la habilidad para interactuar con empatía y compasión.

El mindfulness se ha asociado a un aumento de actitudes empáticas y compasivas. Médicos que puntúan alto para actitudes basadas en mindfulness evidencian mayor comprensión acerca de la experiencia de enfermar, mayor comprensión del contexto, menor reactividad emocional, menor tendencia a juzgar y una más robusta alianza terapéutica con el paciente ${ }^{10,72-74}$.

Así, el mindfulness contribuye a reducir actitudes inconscientemente sesgadas o discriminadoras que pueden llegar a afectar los resultados en salud ${ }^{62(\mathrm{p} .79), 75}$.

\section{Relaciones laborales y conexión institucional}

Un clima institucional desfavorable e infraestructura deficiente han sido identificados como factores de riesgo importante de estrés y burnout en salud $^{76}$. Asimismo, las relaciones laborales pueden ser una de las dimensiones más gratificantes o más frustrantes en el ejercicio de la medicina. El cultivo del mindfulness puede contribuir a desarrollar una cultura institucional con mayor sentido, aun cuando existan limitaciones de recursos e infraestructura. Uno de los efectos del mindfulness es la capacidad de apreciar la "humanidad compartida" inherente a la existencia humana. Esa capacidad conlleva dos dimensiones que interactúan entre sí: la de comprender que la postura propia no es la única y la de valorar el bienestar colectivo tanto como el propio. Existe evidencia que sugiere que los grupos profesionales en salud que favorecen la dimensión colectiva y colaborativa en el trabajo tienden a presentar menor cantidad de errores y a experimentar un mayor sentido institucional ${ }^{77-80}$.

En la siguiente tabla exponemos las seis dimensiones de la práctica médica recién descritas, ejemplificando las dificultades que pueden presentarse en dichas dimensiones y cómo el mindfulness puede contribuir a enfrentarlas (Tabla 1).

\section{Vías para el desarrollo de resiliencia en Medicina}

Se ha señalado que cuidar y cuidarse son dos caras del mismo fenómeno, que se despliegan de modo sinérgico en el camino para promover la resiliencia en medicina ${ }^{62(\mathrm{p} .200)}$. Ha sido elusivo identificar factores y vías a través de las cuales la resiliencia médica puede ser desarrollada ${ }^{3,80}$. Surge, por lo tanto, el desafío de identificar las vías a través de las cuales el entrenamiento en mindfulness, en particular, favorece la calidad del encuentro clínico y la calidad del cuidado personal.

Sugerimos que los distintos ámbitos antes revisados, se agrupan bajo dos ejes que median en su desarrollo y permiten que ocurran.

\section{Eje I: Efecto inespecífico y específico de las IBM}

Consiste en factores comunes que son compartidos por distintas modalidades de intervención que promueven la resiliencia médica. En la dimensión inespecífica es posible identificar la necesidad de cambio personal ${ }^{81}$, la motivación 
Tabla 1. Ejemplos de situaciones clínicas y cómo la práctica médica basada en mindfulness puede contribuir a enfrentarlas

\begin{tabular}{|c|c|c|}
\hline $\begin{array}{l}\text { Dimensión de la } \\
\text { relación clínica }\end{array}$ & Dificultad o situación clínica & $\begin{array}{l}\text { Beneficio de práctica clínica que incorpora } \\
\text { mindfulness }\end{array}$ \\
\hline Comunicación & $\begin{array}{l}\text { El paciente responde a las preguntas } \\
\text { del médico, pero éste sólo atiende a } \\
\text { aspectos de dichas respuestas o realiza } \\
\text { una comprensión superficial de ellas }\end{array}$ & $\begin{array}{l}\text { Se logra mayor atención del proceso comunicativo } \\
\text { Se atiende al correlato paraverbal del paciente y el } \\
\text { propio, reconociendo dimensiones afectivas y nece- } \\
\text { sidades a un nivel más profundo }\end{array}$ \\
\hline $\begin{array}{l}\text { Razonamiento clínico y } \\
\text { toma de decisiones }\end{array}$ & $\begin{array}{l}\text { Pistas, signos y síntomas pueden ser } \\
\text { pasados por alto. Presión por deter- } \\
\text { minar un diagnóstico y resolver una } \\
\text { situación clínica }\end{array}$ & $\begin{array}{l}\text { El mindfulness puede ser visto como una herramienta } \\
\text { clínica, al promover la eficiencia de las funciones } \\
\text { ejecutivas superiores, tales como atención flotante y } \\
\text { dirigida, memoria de trabajo, control de impulsos y } \\
\text { visión en perspectiva. Se tolera mejor el "no saber" }\end{array}$ \\
\hline $\begin{array}{l}\text { Desarrollo de empatía y } \\
\text { compasión }\end{array}$ & $\begin{array}{l}\text { Sesgo social implícito, situaciones clíni- } \\
\text { cas con pacientes difíciles }\end{array}$ & $\begin{array}{l}\text { Se desarrolla la capacidad de estar presente frente } \\
\text { al sufrimiento, tanto con el propio como con el del } \\
\text { paciente } \\
\text { Se desarrolla la comprensión de "humanidad com- } \\
\text { partida", limitando el sesgo social }\end{array}$ \\
\hline Estrés y burnout & $\begin{array}{l}\text { Registros electrónicos, sobrecarga } \\
\text { asistencial }\end{array}$ & $\begin{array}{l}\text { Atender de forma consciente a las señales somáticas } \\
\text { de estrés y revertirlas mediante técnicas meditativas } \\
\text { breves }\end{array}$ \\
\hline Satisfacción del paciente & $\begin{array}{l}\text { No entrega de información suficiente } \\
\text { por prisa }\end{array}$ & $\begin{array}{l}\text { Prestar atención a la necesidad específica de informa- } \\
\text { ción del paciente y poner mayor énfasis en esa área }\end{array}$ \\
\hline $\begin{array}{l}\text { Relaciones laborales y } \\
\text { conexión institucional }\end{array}$ & $\begin{array}{l}\text { Prevención de caídas en una unidad de } \\
\text { hospitalizados }\end{array}$ & $\begin{array}{l}\text { Todos somos humanos; todos poseemos caracterís- } \\
\text { ticas positivas y negativas. La prevención de caídas } \\
\text { es un trabajo colectivo. Si me siento parte de una } \\
\text { comunidad, participaré más activamente en las tareas } \\
\text { comunes, como prevenir caídas }\end{array}$ \\
\hline
\end{tabular}

para el cambio ${ }^{82}$, la identificación de déficit personales $^{83}$ y el componente psicoeducativo de este tipo de intervenciones ${ }^{83}$. El plano específico está determinado por el efecto directo de las prácticas contemplativas sobre las funciones cognitivas y emocionales. Entre estos efectos es posible distinguir habilidades tales como incremento atencional focalizado y flotante ${ }^{84,85}$, la regulación de la tendencia de control cognitivo ${ }^{84}$, el automonitoreo ${ }^{84}$, la tendencia a no enjuiciar, el incremento de la empatía y la compasión ${ }^{72-74,86}$, y la reducción de sesgos cognitivos y sociales ${ }^{75}$. Ambos planos interactúan, posibilitando los beneficios asociados a la práctica del mindfulness en la práctica médica.

\subsection{Eje II: Integración conocimiento implícito y explícito de las IBM}

El conocimiento explícito e implícito (también llamados procesamiento tipo 1 y tipo 2), consisten en dos grandes modos a través de los cuales se realiza el proceso de juicio clínico y de toma de decisiones en Medicina ${ }^{32,88}$. El conocimiento implícito suele ser inconsciente y se apoya sobre la intuición, la experiencia, habilidades, valores y la valoración de los contextos. El conocimiento explicito hace referencia a todo aquel que transita a nivel consciente y que se sustenta sobre el análisis de información cuantificable, basada en la evidencia y que resulta comunicable. Tradicionalmente, el ejercicio de la medicina ha sido asociado al ejercicio del conocimiento explícito. Sin embargo, la evidencia sugiere que los procesos implícitos son ampliamente utilizados en la construcción del juicio clínico y de toma de decisiones $^{31,88}$. Aspectos como ambigüedades clínicas, contextos, valores del paciente, sesgos y prejuicios, emociones o intereses del médico son dimensiones que participan tanto como el raciocinio, la lógica o la evidencia en el proceso de toma de decisiones en Medicina. Una práctica médica basada en 
mindfulness puede favorecer que la ambigüedad de la realidad clínica y la subjetividad inherente a la relación entre médico y paciente puedan ser más conscientes, reduciendo así el riesgo de errores, la tendencia al sesgo cognitivo y social y mejorando el proceso de toma de decisiones. El mindfulness puede actuar como un agente que articula e integra ambos modos de conocimiento ${ }^{32,89}$.

En la Figura 1 se ilustra la articulación entre una Medicina que integra el mindfulness, los beneficios observados de una práctica basada en mindfulness y el desarrollo de la resiliencia médica. Asimismo, se describe como los beneficios observados son mediados por los dos ejes descritos: el eje de los cambios inespecífico y específico logrados a través de las IBM y por el eje de la integración del conocimiento implícito y explícito.

IBM para médicos o estudiantes de Medicina no son la única forma de promover la resiliencia médica. Se han descrito muchas otras formas ${ }^{7-9}$ que han demostrado eficacia para reducir el estrés, mejorar la calidad de atención y aumentar la capacidad empática. Posiblemente, también existe imprecisión al momento de dar cuenta acerca del real alcance de la atención plena, exagerando sobre la universalidad de sus beneficios ${ }^{90}$. El mindfulness aplicado a la práctica médica es un camino, entre otros, y como tal puede ser de utilidad para algunos y para otros puede no serlo.

Existen organizaciones que integran el mindfulness, así como existen experiencias en instituciones en salud que demuestran que existen modelos y valores institucionales que promueven la resiliencia individual y colectiva ${ }^{78,91,92}$.

\section{Conclusiones}

La evidencia sugiere que un proceso clínico de excelencia debe incluir no sólo información anamnésica, de laboratorio o de medicina basada en la evidencia, sino también todos aquellos factores que promueven una relación clínica saludable y un proceso de toma de decisiones coordinado con los valores y preferencias del paciente.

Lograr mayores niveles de resiliencia individual, grupal e institucional es un desafío que incluye a todos los miembros de la fuerza laboral en salud y a las instituciones que los cobijan. Así aumentará la calidad de la atención al mismo tiempo que se reducirán los errores médicos, el estrés y el burnout.

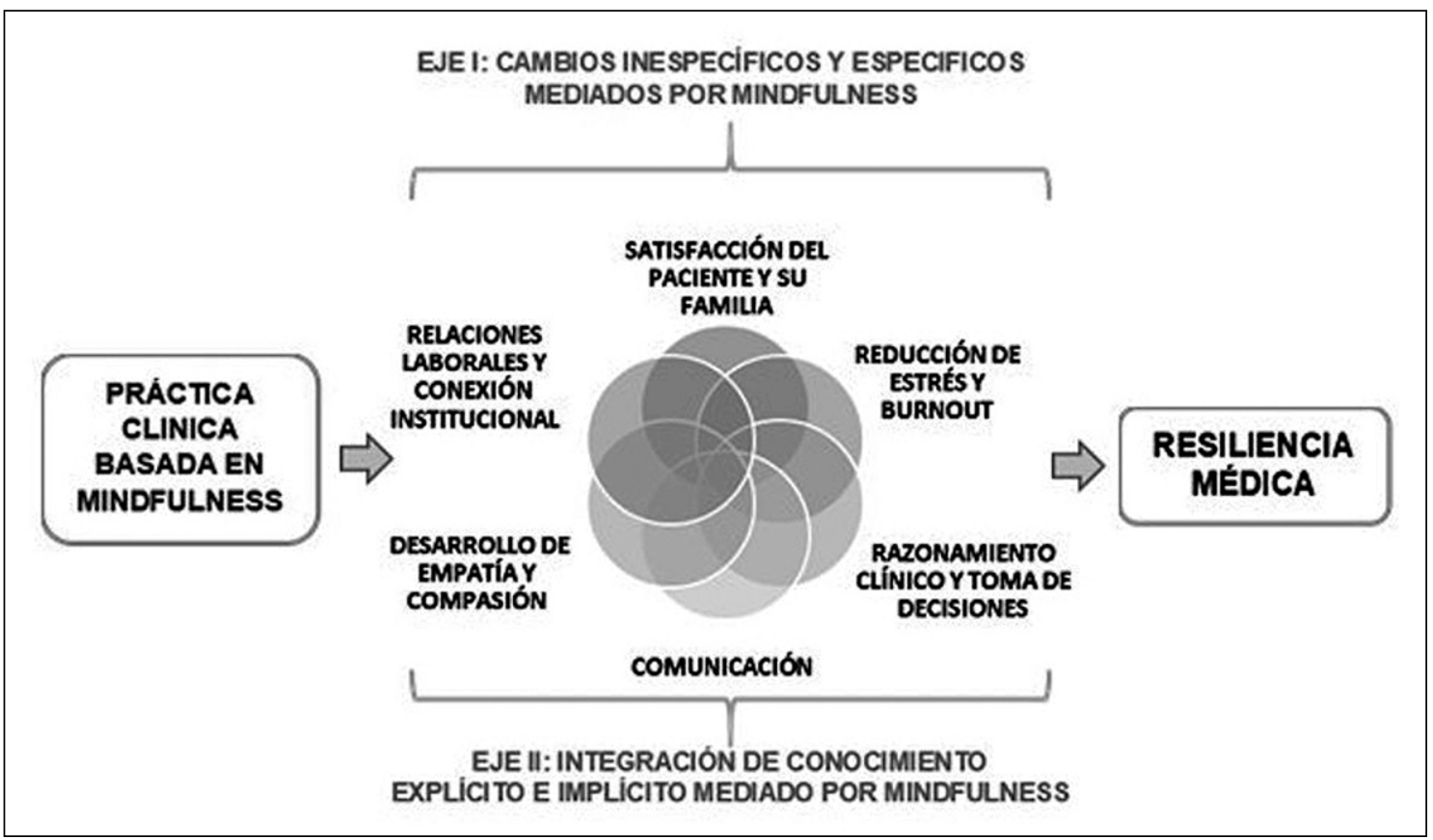

Figura 1. Modelo general de una práctica médica basada en mindfulness. 
El entrenamiento y el cultivo de una práctica médica que incorpora el mindfulness emerge como una vía valiosa para promover la resiliencia en salud. Si bien ya existen experiencias internacionales (Universidades de Rochester, McGill, Monash, Georgetown, etc.) y nacionales (Universidades Adolfo Ibáñez, Austral de Chile, Católica de Chile y del Desarrollo), poder contar con programas de formación continua para profesionales de la salud y modelos de educación transversal en la educación de estudiantes de Medicina y otras carreras de la salud, surge como una un desafío y una oportunidad para lograr una práctica resiliente en salud.

Agradecimientos: Álvaro Langer contó con el apoyo de la Iniciativa Científica Milenio del Ministerio de Economía, Fomento y Turismo, proyecto "Núcleo Milenio para Mejorar la Salud Mental de Adolescentes y Jóvenes, Imhay". Sebastián Medeiros y Álvaro Langer contaron con el apoyo de del Fondo de Innovación para la Competitividad (FIC) del Ministerio de Economía, Fomento y Turismo, a través de la Iniciativa Científica Milenio, ProyectoIS130005 (MIDAP).

\section{Referencias}

1. Fletcher D, Mustafa S. Psychological resilience: A review and critique of definitions, concepts, and theory. Eur Psychol 2013; 18 (19): 12-23.

2. Cheshire A, Hughes J, Lewith G, Panagioti M, Peters D, Simon C, et al. GPs' perceptions of resilience training: a qualitative study. Br J Gen Pract 2017; disponible en: https://bjgp.org [Consultado el 11 de septiembre de 2018].

3. Zwack J, Schweitzer J. If every fifth physician is affected by burnout, what about the other four? Resilience strategies of experienced physicians. Acad Med 2013; 88 (3): 382-9.

4. Epstein RM, Krasner M. Physician resilience: what it means, why it matters, and how to promote it. Acad Med 2013; 88 (3): 301-3.

5. Weng H, Hung C, Liu Y, Cheng Y, Yen C, Chang C, et al. Associations between emotional intelligence and doctor burnout, job satisfaction and patient satisfaction. Med Educ 2011; 45: 835-42.

6. Batbaatar E, Dorjdagva J, Luvsannyam A, Savino MM, Amenta P. Determinants of patient satisfaction: a systematic review. Perspect Public Heal 2017; 137 (2): 89-101.
7. West CP, Dyrbye LN, Rabatin JT, Call TG, Davidson $\mathrm{JH}$, Multari A, et al. Intervention to promote physician well-being, job satisfaction, and professionalism: a randomized clinical trial. JAMA Intern Med 2014; 174 (4): 527-33.

8. Fortney L, Luchterhand Ch, Zakletskaia L, Zgierska A, Rakel D. Abbreviated mindfulness intervention for job satisfaction, quality of life, and compassion in primary care clinicians: a pilot study. Ann Fam Med 2013; 11 (5): 412-20.

9. Fox S, Lydon S, Byrne D, Madden C, Connolly F, O'connor P. A systematic review of interventions to foster physician resilience. Postgrad Med J 2018; 94 (1109): 162-70.

10. Krasner MS, Epstein RM, Beckman H, Suchman AL, Chapman B, Mooney CJ, et al. Association of an educational program in mindful communication with burnout, empathy, and attitudes among primary care physicians. JAMA 2009; 302 (12): 1284-93.

11. Goldhagen BE, Kingsolver K, Stinnett SS, Rosdahl JA. Stress and burnout in residents: impact of mindfulness-based resilience training. Adv Med Educ Pract 2015; 6: 525-32.

12. Goodman MJ, Schorling JB. A mindfulness course decreases burnout and improves well-being among healthcare providers. Intern J Psychiat Med 2012; 43 (2): 119-28.

13. Monteiro LM, Musten RF, Compson J. Traditional and contemporary mindfulness: finding the middle path in the tangle of concerns. Mindfulness 2015; 6 (1): 1-13.

14. Bishop SR, Lau M, Shapiro S, Carlson L, Anderson ND, Carmody J, et al. Mindfulness: A proposed operational definition. Clin Psychol 2004; 11 (3): 230-41.

15. Black DS. A brief definition of mindfulness. Behav Neurosci 2011; 7 (2): 109.

16. Germer C. What is mindfulness. Insight Journal 2004; 22: 24-9.

17. Gotink RA, Chu P, Busschbach JJ, Benson H, Fricchione GL, Hunink MM. Standardised mindfulness-based interventions in healthcare: an overview of systematic reviews and meta-analyses of RCTs. PloSone 2015; 16; 10 (4): e0124344. Disponible en: www.plos.org [Consultado el 12 de septiembre de 2018].

18. Kabat-Zinn J. Some reflections on the origins of MBSR, skillful means, and the trouble with maps. Contemp Buddhism 2011; 12 (01): 281-306.

19. Williams JMG, Kabat-Zinn J. Mindfulness: diverse perspectives on its meaning, origins, and multiple applications at the intersection of science and dharma. Contemp Buddhism 2011; 12 (1): 1-18.

20. Shapiro SL, Carlson LE. The art and science of mind- 
fulness: Integrating mindfulness into psychology and the helping professions (2nd ed.). Washington, DC, US: American Psychological Association. 2017.

21. Hedman-LagerlöfM, Hedman-Lagerlöf E, Öst LG. The empirical support for mindfulness-based interventions for common psychiatric disorders: a systematic review and meta-analysis. Psychol Med 2018; 48 (13): 2116-29.

22. Grossman P, Niemann L, Schmidt S, Walach H. Mindfulness-based stress reduction and health benefits: A meta-analysis. J Psychosom Res 2004; 57 (1): 35-43.

23. Waters L, Barsky A, Ridd A, Allen K. Contemplative education: A systematic, evidence-based review of the effect of meditation interventions in schools. Educ Psychol Rev 2015; 27 (1): 103-34.

24. Eby LT, Allen TD, Kate M, Conley RL, Henderson W, Mancini V. Mindfulness-based training interventions for employees: A qualitative review of the literature. Hum Resour Manage Rev. 2017. Disponible en: www. researchgate.net [Consultado el 14 de septiembre de 2018].

25. De Vibe MF, Bjørndal A, Fattah S, Dyrdal GM, Halland E, Tanner-Smith E. Mindfulness-based stress reduction (MBSR) for improving health, quality of life and social functioning in adults: a systematic review and meta-analysis. Campbell Sysyematic Review 2012; 8 (3): $1-127$.

26. Gotink RA, Chu P, Busschbach J, Benson H, Fricchione GL, Hunink M. Standardised mindfulness-based interventions in healthcare: an overview of systematic reviews and meta-analyses of RCTs. PloSone 2015; 10 (4): e0124344. Disponible en: www.plos.org [Consultado el 2 de octubre de 2018].

27. Dobkin PL, Bernardi N, Bagnis C. Enhancing clinicians' well-being and patient-centered care through mindfulness. J ContinEduc Health 2016; 36 (1): 11-6.

28. Amutio-Kareaga A, García-Campayo J, Delgado LC, Hermosilla D, Martínez-Taboada C. Improving communication between physicians and their patients through mindfulness and compassion-based strategies: a narrative review. J Clin Med 2017; 6 (3): 33. Disponible en: www.mdpi.com [Consultado el 2 de octubre de 2018].

29. Burgess DJ, Beach MC, Saha S. Mindfulness practice: A promising approach to reducing the effects of clinician implicit bias on patients. Pat Educ Couns 2017; 100 (2): 372-6.

30. Burton A, Burgess C, Dean S, Koutsopoulou G, Hugh Jones S. How effective are mindfulness based interventions for reducing stress among healthcare professionals? A systematic review and meta analysis. Stress Health 2017; 33 (1): 3-13.
31. Warnecke E, Ogden K, Bentley M, Nelson M. 5-year follow-up of a randomised controlled trial of the effects of mindfulness practice on medical practitioners' stress. Med Ed Publish 2017; 6 (1). Disponibleenwww.mededpublish.org [Consultado el 3 de octubre de 2018].

32. Epstein RM. Mindful practice. JAMA 1999; 282 (9): 833-9.

33. Dobkin PL, Hassed CS. Mindful medical practitioners. $1^{\text {st }}$ Ed. Geneva, Switzerland: Springer; 2016.

34. clínicaconsciente.cl [internet]. Valdivia: Instituto de Neurociencias Clínicas; c2018 [Citado el 3 de noviembre de 2018]. Disponible en https://www.clinicaconsciente. $\mathrm{cl} /$.

35. Shapiro SL, Carlson LE, Astin JA, Freedman B. Mechanisms of mindfulness. J Clin Psychol 2006; 62 (3): 373-86.

36. Sauer S, Lynch S, Walach H, Kohls N. Dialectics of mindfulness: implications for western medicine. Philos Ethics Humanit Med 2011; 6 (1): 10. Disponible en: www.biomedcentral.com [Consultado el 5 de octubre de 2018].

37. Young KS, van der Velden AM, Craske MG, Pallesen KJ, Fjorback L, Roepstorff A, Parsons CE. The impact of mindfulness-based interventions on brain activity: A systematic review of functional magnetic resonance imaging studies. Neurosci Biobehav Rev 2017; 8: 824-33.

38. Singer T, Engert V. It Matters What You Practice: Differential Training Effects on Subjective Experience, Behavior, Brain and Body in the ReSource Project. Curr Opini Psychol 2018. Disponible en: www.sciencedirect. com [Consultado el 2 de noviembre de 2018].

39. Guendelman S, Medeiros S, Rampes H. Mindfulness and emotion regulation: Insights from neurobiological, psychological, and clinical studies. Front Psychol 2017; 8: 220. Disponible en: www.frontiersin.org [Consultado el 5 de noviembre de 2018].

40. Superintendencia de Salud. Estudio de opinión a usuarios del sistema de salud, reforma y posicionamiento de la superintendencia de salud (Jefa de Proyecto: Paulina Valenzuela A. Contraparte Técnica: Marcela Pezoa G). Marzo de 2015. Disponible en: http://www.supersalud. gob.cl/portal/w3-article-12430.html [Consultado el 6 de noviembre de 2018].

41. Jackson JL, Chamberlin J, Kroenke K. Predictors of patient satisfaction. Soc Sci Med 2001; 52 (4): 609-20.

42. Ha JF, Longnecker N. Doctor-patient communication: a review. Ochsner J 2010; 10 (1): 38-43.

43. Williams SJ, Calnan M. Key determinants of consumer satisfaction with general practice. Family practice 1991; 8 (3): 237-42.

44. Krogh E, Langer AI, Schmidt C. Therapeutic Presence 
and its contribution to the doctor-patient encounter. J Contin Educ Health Prof 2019; 39 (1): 49-53.

45. DiMatteo MR, Taranta A, Friedman HS, Prince LM. Predicting patient satisfaction from physicians' nonverbal communication skills. Med Care 1980: 376-87.

46. Kee JW, Khoo HS, Lim I, Koh MY. Communication Skills in Patient-Doctor Interactions: Learning from Patient Complaints. Health Prof Educ 2018; 4 (2): 97106.

47. Zolnierek KB, DiMatteo MR. Physician communication and patient adherence to treatment: a meta-analysis. Med Care 2009; 47 (8): 826-34.

48. Epstein RM, Fiscella K, Lesser CS, Stange KC. Why the nation needs a policy push on patient-centered health care. Health affairs 2010; 29 (8): 1489-95.

49. Rodrigues H, Cobucci R, Oliveira A, Cabral JV, Medeiros L, Gurgel $\mathrm{K}$, et al. Burnout syndrome among medical residents: A systematic review and meta-analysis. PloSone 2018; 13 (11): e0206840. Disponible en www.plos.org [Consultado el 6 de noviembre de 2018].

50. Shanafelt TD, Hasan O, Dyrbye LN, Sinsky C, Satele $\mathrm{D}$, Sloan J, et al. Changes in burnout and satisfaction with work-life balance in physicians and the general US working population between 2011 and 2014. Mayo Clin Proc 2015; 90 (12): 1600-13.

51. West CP, Dyrbye LN, Shanafelt TD. Physician burnout: contributors, consequences and solutions. J Int Med 2018; 283 (6): 516-29.

52. Dewa CS, Loong D, Bonato S, Trojanowski L. The relationship between physician burnout and quality of healthcare in terms of safety and acceptability: a systematic review. Br Med J 2017; 7 (6): e015141. Disponible en: www.bmj.com [Consultado el 9 de noviembre de 2018].

53. Lee RT, Seo B, Hladkyj S, Lovell BL, Schwartzmann L. Correlates of physician burnout across regions and specialties: a meta-analysis. Hum Res Health 2013; 11 (1): 48. Disponible en: www.biomedcentral.com [Consultado el 9 de noviembre de 2018].

54. West CP, Dyrbye LN, Erwin PJ, Shanafelt TD. Interventions to prevent and reduce physician burnout: a systematic review and meta-analysis. Lancet 2016; 388 (10057): 2272-81.

55. Panagioti M, Panagopoulou E, Bower P, Lewith G, Kontopantelis E, Chew-Graham C, et al. Controlled interventions to reduce burnout in physicians: a systematic review and meta-analysis. JAMA Int Med 2017; 177 (2): 195-205.

56. Daya Z, Hearn JH. Mindfulness interventions in medical education: A systematic review of their impact on medical student stress, depression, fatigue and burnout. Med Teach 2018; 40 (2): 146-53.
57. Luken M, Sammons A. Systematic review of mindfulness practice for reducing job burnout. Am J Occup Ther 2016; 70 (2): 1-7.

58. Medeiros S, Pulido R. Programa de Reducción de Estrés basado en Mindfulness para funcionarios de la salud: experiencia piloto en un hospital público de Santiago de Chile. Rev Chil Neuro-Psiquiat 2011; 49 (3): 251-7.

59. Brito G. Programa de reducción del estrés basado en la atención plena (mindfulness): sistematización de una experiencia de su aplicación en un hospital público semi-rural del sur de Chile. Psicoperspectivas 2011; 10 (1): 221-42.

60. West CP, Dyrbye LN, Rabatin JT, Call TG, Davidson $\mathrm{JH}$, Multari A, et al. Intervention to promote physician well-being, job satisfaction, and professionalism: a randomized clinical trial. JAMA Int Med 2014; 174 (4): 527-33.

61. Croskerry P, Singhal G, Mamede S. Cognitive debiasing 1: origins of bias and theory of debiasing. BMJ Qual Saf 2013; 22: 58-64.

62. Epstein R. Attending: medicine, mindfulness, and humanity. New York, NY. $1^{\text {th }}$. Ed.: Simon and Schuster; 2017.

63. Borrell-Carrió F, Epstein RM. Preventing errors in clinical practice: a call for self-awareness. Ann Fam Med 2004; 2 (4): 310-6.

64. Sorenson C, Bolick B, Wright K, Hamilton R. Understanding compassion fatigue in healthcare providers: A review of current literature. J Nurs Scholarsh 2016; 48 (5): 456-65.

65. Sinclair S, Raffin-Bouchal S, Venturato L, Mijovic-Kondejewski J, Smith-MacDonald L. Compassion fatigue: A meta-narrative review of the healthcare literature. Int J Nurs Stud 2017; 69: 9-24.

66. Newell JM, MacNeil GA. Professional burnout, vicarious trauma, secondary traumatic stress, and compassion fatigue. Best Pract Ment Health 2010; 6 (2): 57-68.

67. Gleichgerrcht E, Decety J. The relationship between different facets of empathy, pain perception and compassion fatigue among physicians. Front BehavNeurosci8 (2014): 243. Disponible en: www.frontiersin.org [Consultado el 12 de noviembre de 2018].

68. Injeyan MC, Shuman C, Shugar A, Chitayat D, Atenafu EG, Kaiser A. Personality traits associated with genetic counselor compassion fatigue: The roles of dispositional optimism and locus of control. J Gen Couns 2011; 20 (5): 526-40.

69. van Mol MM, Kompanje EJ, Benoit DD, Bakker J, Nijkamp MD. The prevalence of compassion fatigue and burnout among healthcare professionals in intensive 
care units: a systematic review. PloSone. 2015; 31; 10 (8): e0136955. Disponible en www.plos.org [Consultado el 12 de noviembre de 2018].

70. Dugani S, Afari H, Hirschhorn LR, Ratcliffe H, Veillard J, Martin G, Lagomarsino G, Basu L, Bitton A. Prevalence and factors associated with burnout among frontline primary health care providers in low-and middle-income countries: A systematic review. Gates Open Res 2018; 11; 2. Disponible en: www.gatesopenresearch.com [Consultado el 12 de noviembre de 2018].

71. Lupien SJ, McEwen BS, Gunnar MR, Heim C. Effects of stress throughout the lifespan on the brain, behaviour and cognition. Nat Rev Neurosci 2009; 10 (6): 434-45.

72. Beckman HB, Wendland M, Mooney C, Krasner MS, Quill TE, Suchman AL, et al. The impact of a program in mindful communication on primary care physicians. Acad Med 2012; 1; 87 (6): 815-9.

73. Beach MC, Roter D, Korthuis PT, Epstein RM, Sharp V, Ratanawongsa N, et al. A multicenter study of physician mindfulness and health care quality. Ann Fam Med 2013; 11 (5): 421-8.

74. Dobkin PL, Bernardi NF, Bagnis CI. Enhancing clinicians' well-being and patient-centered care through mindfulness. J Cont Educe Health Prof 2016; 36 (1): 11-6.

75. Hall WJ, Chapman MV, Lee KM, Merino YM, Thomas TW, Payne BK, et al. Implicit racial/ethnic bias among health care professionals and its influence on health care outcomes: a systematic review. Am J Pub Health 2015; 105 (12): 60-76.

76. Montgomery A, Todorova I, Baban A, Panagopoulou E. Improving quality and safety in the hospital: The link between organizational culture, burnout, and quality of care. Br J Health Psychol 2013; 18 (3): 656-62.

77. Singh NN, Singh SD, Sabaawi M, Myers RE, Wahler RG. Enhancing treatment team process through mindfulness-based mentoring in an inpatient psychiatric hospital. Behav Mod 2006; 30 (4): 423-41.

78. Good DJ, Lyddy CJ, Glomb TM, Bono JE, Brown KW, Duffy MK, et al. Contemplating mindfulness at work: An integrative review. J Manag 2016; 42 (1): 114-42.

79. Vogus TJ, Sutcliffe KM. The impact of safety organizing, trusted leadership, and care pathways on reported medication errors in hospital nursing units. Med Care 2007;
1: 997-1002.

80. Suchman AL, Sluyter DJ, Williamson PR, editors. Leading change in healthcare: transforming organizations using complexity, positive psychology and relationship-centered care. London. Radcliffe Publishing; 2011.

81. Seligman ME, Steen TA, Park N, Peterson C. Positive psychology progress: empirical validation of interventions. Am Psychol 2005; 60 (5): 410-25.

82. Lyubomirsky S, Dickerhoof R, Boehm JK, Sheldon KM. Becoming happier takes both a will and a proper way: An experimental longitudinal intervention to boost well-being. Emotion 2011; 11 (2): 391-402.

83. Michie S, Van Stralen MM, West R. The behaviour change wheel: a new method for characterising and designing behaviour change interventions. ImplementSci. 2011; 6 (1): 42. Disponible en: www.biomedcentral.com [Consultado el 20 de noviembre de 2018].

84. Lutz A, Slagter HA, Dunne JD, Davidson RJ. Attention regulation and monitoring in meditation. Trends Cogn Sci 2008; 12 (4): 163-9.

85. Brown KW, Ryan RM. The benefits of being present: mindfulness and its role in psychological well-being. J Pers Soc Psychol 2003; 84 (4): 822-844.

86. Raab K. Mindfulness, self-compassion, and empathy among health care professionals: a review of the literature. J Health Care Chaplain 2014; 20 (3): 95-108.

87. Malterud K. The art and science of clinical knowledge: evidence beyond measures and numbers. Lancet 2001; 358 (9279): 397-400.

88. Goldman GM. The tacit dimension of clinical judgment. Yale J Biol Med 1990; 63 (1): 47-61.

89. Epstein RM. Mindful practice in action (I): Technical competence, evidence-based medicine, and relationship-centered care. Fam Syst Health 2003; 21 (1): 1-9.

90. Van Dam NT, van Vugt MK, Vago DR, Schmalzl L, Saron CD, Olendzki A, et al. Mind the hype: A critical evaluation and prescriptive agenda for research on mindfulness and meditation. Perspect Psychol Sci 2018; 13 (1): 36-61.

91. Carthey J, de Leval M, Reason J. Institutional resilience in healthcare systems. BMJ Qual Saf 2001; 10 (1): 29-32.

92. Vogus TJ, Sutcliffe KM. Organizational mindfulness and mindful organizing: A reconciliation and path forward. Acad Manag Learn Edu 2012; 11 (4): 722-35. 